\section{Response to Suthers and Mina}

We thank Suthers and Mina ${ }^{1}$ for their interest in our paper. ${ }^{2}$ While we have common ground with them on some points, there are others on which we disagree or that need clarification. Overall, we stand by our approach and conclusions, which are supported by the results of our study.

As noted by Suthers and Mina, we appropriately restricted our analysis to genes with a known associated Mendelian phenotype. It would be impossible to do otherwise, because any report issued in the course of a screening program must relate to a specific disease risk. With regard to allele frequencies, we filtered to exclude common variation (which is unlikely to be pathogenic) as is standard practice. We agree in retrospect that a population allele frequency cut-off of 0.01 (carrier frequency of 1 in 50) is probably too stringent, and in recent work we have relaxed that to 0.02 , with the common CFTR variant p.Phe508del informatically excluded from the cut-off. Our subjects predominantly originated from countries in the Middle East and it is unlikely that this filtering strategy led to us missing any carrier couples. Cystic fibrosis is relatively uncommon in this region, and has a different allele distribution from that in Europe, with p.Phe508del accounting for a minority of disease alleles. ${ }^{3}$ As stated in the paper, we did not include copy-number variant detection in our protocol, so $a$-thalassemia deletions would not have been detected regardless of this consideration. In contrast, we do not understand the criticism of our decision to exclude variants seen in three or more unrelated couples. This would equate to an allele frequency of 0.1, which we regard as very conservative. Even in a population with a high degree of endogamy, such a high allele frequency for a severe condition would be very unusual.

Although we did not specify a gene panel for the purpose of this research study, we recognize that there are some potential advantages to specifying in advance which genes are being analyzed. Certainly, clearly defined criteria for reporting (relating to severity and potential treatability of the included conditions and of specific variants, where information is available) are essential. However, the difficulty with any predefined panel is the very large number of genes that could be included, and the rapid pace of gene discovery. Typical commercially available panels are limited to a few hundred genes-it is noteworthy that three of the four variants we identified were in genes not included in any currently available panel. Even a very large panel will, by excluding some genes, result in carrier couples being missed. The problem is compounded by the fact that countries like Australia have ethnically diverse populations, which means that any attempt to identify the most common few hundred conditions is likely to be relevant for only a subset of couples who might access the test.
Thus, we argue that exome or genome sequencing allow for comprehensiveness and flexibility that is not possible with a limited panel. At population level, there is every reason to be as comprehensive as possible-particularly as the costs of exome sequencing and panels converge.

The flexibility of a genome-wide assay is its principle advantage over even a very large panel. A review of the OMIM database for autosomal recessive and X-linked conditions was conducted in 2016 and repeated in 2018 using the same methodology. An additional 468 genes were identified in 2018 for which a relationship between gene and phenotype had been discovered between 2016 and 2018 (Royston Ong and Nigel Laing, personal communication 2018). While some of these associations may not prove robust, many will be. Redesign and revalidation of a panel on an annual basis is possible, but difficult, and not something that is done by most laboratories. By contrast, adding genes to a list for filtering from exome or genome data is easily accomplished.

Taking these considerations into account, we stand by our view that exome sequencing is the best current option for reproductive carrier screening. It is likely that in the near future, genome sequencing will become sufficiently affordable to allow its use for this purpose at population scale, bringing with it the advantages of more uniform coverage and better copy-number variant detection.

\section{DISCLOSURE}

The authors declare no conflicts of interest.

\section{Edwin P. Kirk, MBBS PhD $D^{1,2,3}$, \\ Kristine Barlow-Stewart, PhD FHGSA, Sarah Josephi-Taylor, MBBS BSc ${ }^{1,3}$ and Tony Roscioli, PhD FRACP ${ }^{1,2,5}$}

${ }^{1}$ Centre for Clinical Genetics, Sydney Children's Hospital, High St, Randwick, NSW, Australia; ${ }^{2}$ Genetics Laboratory, NSW Health Pathology East, Randwick, NSW, Australia; ${ }^{3}$ School of Women's and Children's Health, UNSW Medicine, Randwick, NSW, Australia; ${ }^{4}$ Sydney Medical School Northern, University of Sydney, Royal North Shore Hospital, St Leonards, NSW, Australia; ${ }^{5}$ Neuroscience Research Australia, Randwick, NSW, Australia. Correspondence: Edwin P. Kirk (edwin.kirk@health.nsw.gov.au)

\section{REFERENCES}

1. Suthers G, Mina K. Carrier screening "within the panel". Genet Med. 2018 October; https://doi.org/10.1038/s41436-018-0317-9. [Epub ahead of print].

2. Kirk EP, Barlow-Stewart K, Selvanathan A, et al. Beyond the panel: preconception screening in consanguineous couples using the TruSight One "clinical exome". Genet Med 2018; https://doi.org/10.1038/s41436018-0082-9. [Epub ahead of print].

3. Banjar H, Angyalosi $\mathrm{G}$. The road for survival improvement of cystic fibrosis patients in Arab countries. Int J Pediatr Adol Med. 2015;2:47-58.

doi:10.1038/s41436-018-0318-8 